negative predictive values (PPV, NPV) and area under the ROC curve (AUC).

Results In our cohort of 105 patients, 18 (17\%) had an LVO. VAN was found to have a sensitivity of 0.56 , specificity of 0.77 , PPV of 0.33 , and NPV of 0.89 in predicting LVO, with an AUC of 0.663 . RACE demonstrated a sensitivity of 0.67 , specificity of 0.79 , PPV of 0.40 , and NPV of 0.92 , with an AUC of 0.730 . In patients with time since last known well $(\mathrm{LKW})$ of $\leq 1.5$ hours $(\mathrm{n}=42)$, VAN and RACE both demonstrated superior performance and high NPV (VAN NPV = 0.93, RACE NPV = 0.96).

Conclusions Both VAN and RACE demonstrated measurable but limited benefit in predicting LVO in the field. In patients with LKW times of $\leq 1.5$ hours, RACE showed a high NPV of 0.96. In this subset of patients, RACE could be used to rule out LVO in the prehospital setting, avoiding delays in care due to unnecessary transport to a thrombectomy-capable facility.

Disclosures J. Hosny: None. J. Lynch: None. A. De Havenon: None. M. Tanweer: None. M. Alahmad: None. O. Zaidat: None.

\section{E-058 CAROTID ARTERY STENTING USING BALLOON-GUIDE CATHETER FOR PROXIMAL OCCLUSION AS DISTAL EMBOLIC PROTECTION}

${ }^{1} \mathrm{M}$ Nahhas*, ${ }^{1} \mathrm{G}$ Meeks, ${ }^{1} \mathrm{G}$ Spiegel, ${ }^{1} \mathrm{Y}$ Alderazi, ${ }^{2} \mathrm{M}$ Dannenbaum, ${ }^{1} \mathrm{~S}$ Sheth. ${ }^{1} T h e$ Department of Neurology at McGovern Medical School, The University of Texas Health Science Center at Houston, Houston, TX; ${ }^{2}$ The Department of Neurosurgery at McGovern Medical School, The University of Texas Health Science Center at Houston, Houston, TX

\subsection{6/neurintsurg-2021-SNIS. 153}

Background Distal embolic protection is an important and required component of carotid artery stenting (CAS). Data around distal protection devices (DPD), however, are limited. Here, we evaluate the safety and efficacy of proximal occlusion using balloon guide catheter (BGC) as the primary method of distal embolic protection during CAS.

Methods We conducted a retrospective review of patients undergoing CAS at our healthcare system between January of 2018 to January of 2021. We included patients who received CAS with a balloon-guide catheter being utilized for embolic protection through the inflation of its balloon proximal to the stenotic portion of the cervical internal carotid artery. Following angioplasty and stent deployment, aspiration through its working lumen was performed prior to balloon deflation, for distal embolic protection using flow reversal. Symptomatic patients with emergent CAS were defined as patients receiving CAS in $<24$ hours of presentation for ischemic stroke or TIA ipsilateral to the disease location. Periprocedural ischemic stroke was defined as acute focal neurological symptoms lasting for $\geq 24$ hours following the procedure that was consistent with focal cerebral ischemia ipsilateral to CAS side.

Results A total of 91 CAS procedures were performed during the study period on 87 eligible patients (age 45-93 years), and $65 \%(n=57)$ were males. All procedures were done under proximal BGC protection. In 24 (26.3\%) of cases, a DPD was used in conjunction with the BGC. Severe carotid stenosis cases (as per NASCET criteria) was present in $80.2 \%(n=73)$ of patients. Non-emergent CAS for symptomatic carotid disease accounted for $61.5 \%$ $(n=56)$, while emergent CAS placement for symptomatic carotid disease was $28.6 \% \quad(n=26)$. The remaining $9.9 \%$ $(n=9)$ CAS patients were asymptomatic. Balloon angioplasty was performed in $95.6 \%(\mathrm{n}=87)$ cases with $41.7 \%$ $(\mathrm{n}=38)$ receiving pre-stenting angioplasty, $15.4 \% \quad(\mathrm{n}=14)$ receiving post-stenting angioplasty, and $38.5 \% \quad(\mathrm{n}=35)$ receiving both pre and post stenting angioplasty of the total number of performed procedures. Periprocedural ischemic strokes complication were identified in $3.3 \%$ $(n=3)$ of all cases.

Conclusion Our study indicates a low periprocedural ischemic stroke development following the use of proximal occlusion as the distal embolic protection strategy with BGC in CAS.

Disclosures M. Nahhas: None. G. Meeks: None. G. Spiegel: None. Y. Alderazi: None. M. Dannenbaum: None. S. Sheth: None.

\section{E-059 EPTIFIBATIDE BRIDGING THERAPY FOR STAGED CAROTID ARTERY STENTING AND CARDIAC SURGERY}

M Caton*, K Narsinh, A Baker, J Rapp, W Gasper, J lannuzzi, E Tseng, M Amans, S Hetts, D Cooke. University of California San Francisco, San Francisco, CA

\subsection{6/neurintsurg-2021-SNIS. 154}

Background Prophylactic carotid artery stenting (CAS) is an effective strategy to reduce perioperative stroke in patients with severe carotid stenosis who require cardiothoracic surgery (CTS). Staging both procedures (CAS-CTS) during a single hospitalization presents conflicting demands for antiplatelet therapy and the optimal pharmacologic strategy between procedures is not established. Eptifibatide (Integrellin), a parenteral GP IIb/IIIa antiplatelet agent with a half-life of 2.5 hours, is an appealing alternative to oral thienopyridines, enabling rapid reversal prior to sternotomy. The purpose of this study is to present our initial experience with a "bridging" eptifibatide protocol for staged CAS-CTS.

Methods A retrospective review of staged CAS-CTS procedures at a single referral center was performed. All patients had multivessel coronary and/or valvular disease and severe carotid stenosis $(>70 \%)$ confirmed during preoperative risk assessment. Per the institutional protocol (figure 1), all patients not previously on aspirin were also started on aspirin prior to surgery, followed by eptifibatide during CAS (intraprocedural bolus followed by post-procedural infusion which was continued until the morning of surgery). Pre- and perioperative (30 days) neurologic morbidity and mortality was the primary endpoint.

Results 11 CAS procedures were performed in ten patients using the protocol. The median duration of eptifibatide bridge therapy was 36 hours (range 24-288hrs). There was one minor bleeding complication $(1 / 11,9.1 \%)$ and no major bleeding complications during the bridging and post-operative period. There was one post-operative, non-neurologic death and zero perioperative ischemic strokes.

Conclusions For patients undergoing staged CAS-CTS, Eptifibatide bridging therapy is a viable temporary antiplatelet strategy with a favorable safety profile. This strategy enables a flexible range of time-intervals between procedures. 